\title{
What is the prevalence of rheumatic disorders in general medical inpatients?
}

\author{
C Hood, J Johnson, C Kelly
}

\begin{abstract}
It has been suggested that rheumatological disorders are underdiagnosed in patients with medical problems and that this might be rectified by incorporating a standard brief screening examination as part of the routine assessment of all patients admitted to hospital with medical conditions. Therefore the GALS screening examination was used to assess the prevalence of rheumatic disease in 100 patients admitted with acute medical problems and in a further 100 in the rehabilitative phase of their disease. The nature of locomotor dysfunction in all patients with a positive result was defined by an independent review and then sensitivity and specificity of the screening test was calculated for rheumatic disease in both populations.
\end{abstract}

The median age of the two populations were 63 and 78 years respectively, with more females in the rehabilitation group. The overall prevalence of a positive screening test was $53 \%$ in the acute and $\mathbf{9 4 \%}$ in the chronic disease groups, although the false positive rate in the rehabilitation patients was $30 \%$ due to factors other than rheumatic disorders limiting locomotor function (mainly orthopaedic and neurological conditions). The diagnosis of a rheumatological disorder was made de novo in a significant minority $(10 \%)$ of patients and was usually amenable to treatment. The commonest rheumatic disorder was osteoarthritis which accounted for $55 \%$ of all rheumatic disease, followed by inflammatory joint disease $(16 \%)$, and osteoporosis $(12 \%)$. In addition to osteoporosis, Paget's disease of bone and polymyalgia rheumatica were found more frequently in those patients undergoing rehabilitation than in those admitted with an acute medical problem. A number of clinically important associations between medical and rheumatic disorders were found, such as stroke disease with shoulder capsulitis and heart failure with gout.

The sensitivity of the GALS screening test was extremely high ( $92 \%$ and $100 \%)$, while its specificity fell in the rehabilitation group from $83 \%$ to $17 \%$. None the less, it is felt that this study indicates that the routine use of this test should be considered as part of the assessment of all hospitalised patients with medical problems, whether acute or chronic.

(Postgrad Med f 2001;77:774-777)
Keywords: rheumatic disease; general internal medicine; GALS screening test

Rheumatic disease accounts for more lost working days than any other group of disorders, ${ }^{1}$ and contributes most to the burden of disability in the longer term. ${ }^{2}$ Relatively little is known about the burden of rheumatic disease in an undifferentiated medical inpatient population, although one previous study has estimated the prevalence of rheumatic disease among medical in patients at around $20 \% .^{3}$ However, awareness of rheumatological disorders among junior medical staff can be low, ${ }^{4}$ and many consultant rheumatologists are not involved in the acute general medical rota, thereby denying them the opportunity to educate their juniors in the diagnosis and management of rheumatic disease in the acute setting.

Our aims were first to estimate the prevalence of locomotor disorders in medical inpatients in two contrasting settings in order to assess the contribution of rheumatic disease. Next we wished to assess the distribution of undiagnosed rheumatic disease in the hope that this might be amenable to treatment, and finally we wanted to calculate the sensitivity and specificity of a standard screening examination (GALS) ${ }^{5}$ across an age spectrum.

\section{Methods}

We used a short standardised screening history and examination of the locomotor system that has been shown to be a sensitive indicator of locomotor abnormality ${ }^{6}$ to assess the presence of rheumatic disease. This effectively assesses Gait, together with function in the Arms, Legs, and Spine (GALS). We used this technique to estimate the prevalence of rheumatic disorders in two different groups of medical inpatients. The first group comprised 100 consecutive patients admitted to an acute medical assessment unit and the second comprised 100 patients on a long stay rehabilitation ward for the elderly. The findings at the screening examination were then validated by a consultant rheumatologist who conducted a formal examination of the locomotor system in all patients with a positive screening test. A brief review of GALS negative patients was performed to permit the calculation of the test's sensitivity.

The cause for all abnormalities was defined and was divided into one of three groups: rheumatological, orthopaedic, or locomotor problems due to dysfunction in other organ systems.

We defined rheumatic disorders as any primary disease of the locomotor system developing spontaneously or as a result of drug 
therapy. Orthopaedic conditions were interpreted as locomotor problems due to trauma or requiring surgery, while dysfunction in other organ systems usually arose from disorders of neural tissue or from general frailty and were referred to as "locomotor negative".

The medical notes were accessed to see if any previous locomotor diagnosis had been made and to obtain the results of relevant investigations. Past or present treatment of rheumatic disease was recorded and further tests were then ordered only if they were felt to be necessary to confirm the clinical diagnosis. Further therapeutic suggestions were made when this

A Acute

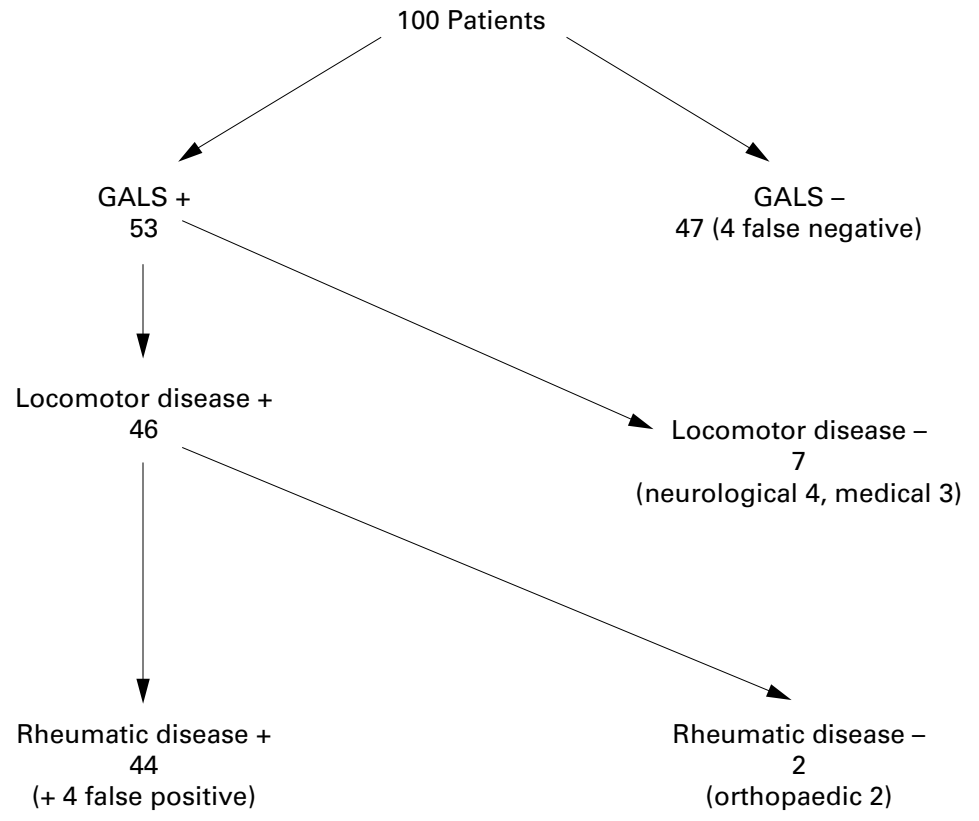

B Chronic

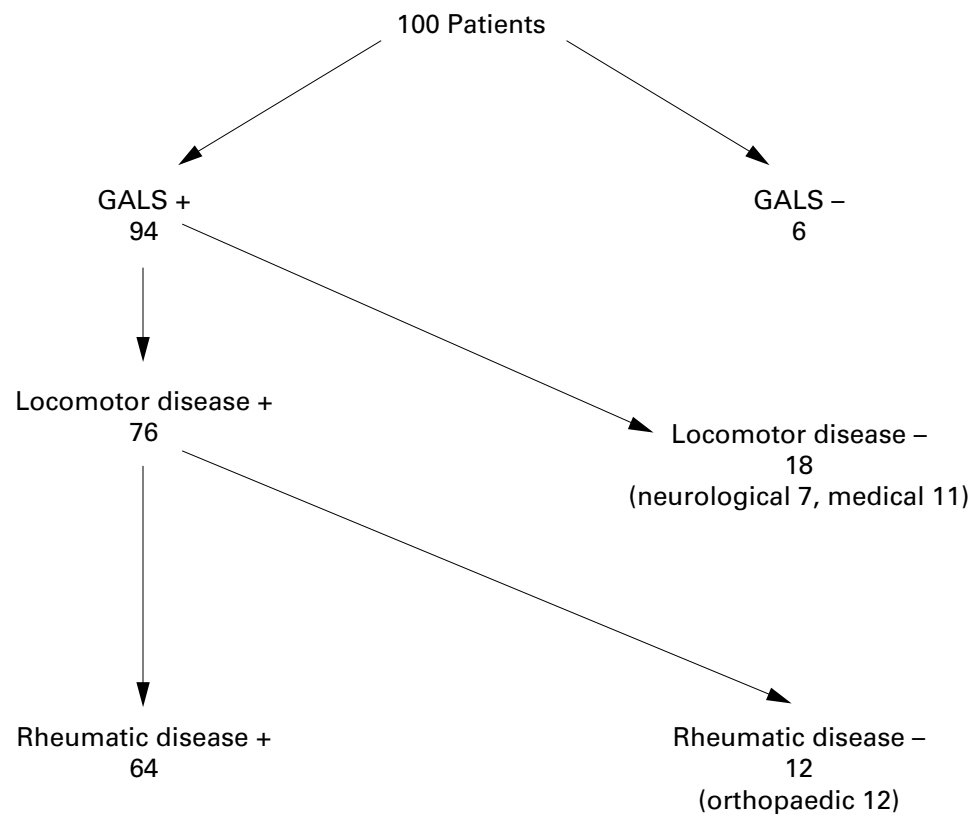

Figure 1 The findings of the GALS screening test in 100 patients admitted to hospital with $(A)$ acute and $(B)$ chronic medical conditions. was felt likely to influence outcome. The specificity, sensitivity, positive and negative predictive values of GALS was then calculated for both groups of patients.

\section{Results}

We found that the median age of the acute admissions was 63 (range 34-89 years) with 53 men, and that the median age of the long stay patients was 78 (range 54-99 years) with 41 men. The GALS screening test was positive in $53 \%$ of acute admissions and $94 \%$ of long stay patients. In addition, four of the acute admissions had some evidence of rheumatic disease but had a negative screening test. These patients had gout (three) or rheumatoid arthritis (one) which was quiescent at the time of admission. Among those with a positive test, rheumatic disease accounted for the majority of cases as shown in fig 1 . Among acute admissions, $9 \%$ had a false positive result because of orthopaedic (two), neurological (four), or other medical conditions (three). In the long stay rehabilitation patients, the false positive rate was $30 \%$, with 12 orthopaedic, seven neurological, and 11 other medical conditions responsible.

Within the acute admissions, 48 patients therefore had rheumatological disease. This had been previously diagnosed in the majority, but constituted a new diagnosis in a significant minority of 15 individuals. Osteoarthritis was the most frequent diagnosis, with inflammatory joint disease, vertebral osteoporosis, disc disease with or without radiculopathy, shoulder capsulitis, and gout all featured significantly. There was no difference in the spectrum of diagnosis between those patients with a newly diagnosed or established rheumatic disease, although neither patient with capsulitis had been previously diagnosed. Among the elderly hospitalised population, there were 64 patients with rheumatic disease. This constituted a new diagnosis in only five cases. The spread of diagnoses were broadly similar to that in the younger group, although osteoarthritis, vertebral osteoporosis, polymyalgia rheumatica, and Paget's disease of the bone were all seen more frequently in the older population. The rheumatological diagnoses within each group are shown in table 1. Patients with osteoarthritis constituted $50 \%$ of rheumatic disease in the younger and $60 \%$ in the older group. The knees were affected in many of these patients and rupture of an associated Baker's cyst leading to a suspicion of deep vein thrombosis had been the reason for admission in three cases. Ultrasonography had confirmed the correct diagnosis in each case. However, if the patients with osteoarthritis are omitted, the prevalence of rheumatic disease in our two populations are $24 \%$ and $26 \%$ respectively.

The finding of a significant burden of rheumatic disease had an impact on treatment. New therapeutic options were offered to all patients with a new diagnosis (20 in total) and a change in therapeutic approach was suggested in seven other patients with established disease. Hence, a significant minority of patients with rheumatic disease ( 27 from a total 
Table 1 The rheumatic disorders identified in 100 patients with (A) acute and (B) chronic medical disorders requiring hospital admission

\begin{tabular}{lrc}
\hline & (A) Acute & (B) Chronic \\
\hline Osteoarthritis & 24 & 38 \\
Inflammatory arthritis & 10 & 6 \\
Osteoporosis & 4 & 9 \\
Symptomatic disc disease & 6 & 0 \\
Gout & 1 & 4 \\
Shoulder capsulitis & 2 & 2 \\
Polymyalgia rheumatica & 0 & 3 \\
Paget's disease & 0 & 2 \\
Systemic lupus & 1 & 0 \\
Total & 48 & 64
\end{tabular}

of $112=24 \%$ ) had a disorder which we felt was amenable to further treatment and therapy was initiated before discharge in all patients who agreed to the proposed intervention.

When the two groups were combined, several associations were noted between rheumatological and medical diagnoses. Most patients with gout had ischaemic heart disease and were on diuretics $(4 / 5)$. Shoulder capsulitis occurred in patients with diabetes $(2 / 4)$ or a previous ipsilateral hemiparesis (2/4). Osteoporosis was associated with long term oral steroid therapy in asthma (3/13) or inflammatory bowel disease (2/13). Patients with rheumatoid arthritis had a high prevalence of diagnosed hypothyroidism (6/14). In addition, patients with orthopaedic causes for a positive screening test (usually a fracture or dislocation) tended to have a high prevalence of clinical and radiological osteoporosis (11/14). Bone mineral densities were later confirmed to be reduced in all 11 of these patients as well as in all cases with osteoporosis of the vertebral spine, where a dorsal kyphosis and reduced spinal movement were invariably present. Primary or secondary prophylaxis had been initiated before this study in only seven of these 24 cases, and was therefore recommended to the remainder as a result of our findings.

The sensitivity of the GALS screening test was found to be $92 \%$ in the acute admissions and $100 \%$ in the long stay patients. However, its specificity fell from $83 \%$ in acute patients to only $17 \%$ in the chronic setting. This represented the coexistence of other pathology in the hospitalised elderly which contributed to their poor mobility. In addition to the orthopaedic causes already described, other conditions associated with a positive screening test included stroke disease and multiple sclerosis. The positive predictive values for the screening test were $83 \%$ and $68 \%$ in the acute and chronic settings, with negative predictive values of $91 \%$ and $100 \%$ respectively.

\section{Discussion}

The GALS test is now an accepted part of the standard assessment of patients who may have rheumatic disease, ${ }^{7}$ and has been recommended for inclusion in the undergraduate teaching program. ${ }^{8}$ The prevalence of rheumatic disease reported in the present study was higher than that previously found for both the patient groups. Several reasons may contribute to this. The median age of our patients was higher than that in a study using the same screening test, ${ }^{3}$ and the high number of patients with osteoarthritis doubled the prevalence of rheumatic disease in our population. Even allowing for this by excluding patients with osteoarthritis, about a quarter of all patients had evidence of a rheumatic disorder. Other factors may also account for this difference as we screened consecutive patients and included all positive results, even if the degree of disease was not likely to affect mobility. However, our results are, if anything, still an underestimate of the true prevalence of rheumatic disease as the consultant only fully examined those patients with a positive GALS test. Clearly the prevalence of locomotor disease in general, and rheumatic disorders in particular, is greatest in an elderly inpatient population. We estimated that a significant percentage of these individuals were still in hospital at the time of this survey as a direct consequence of their immobility.

Osteoarthritis, especially of the knees, was the most frequent diagnosis in both patient groups, but was especially common in the elderly population. Judicious use of intra-articular steroids, combined with analgesia and physiotherapy was employed, especially in those with a ruptured Baker's cyst. Mechanical back pain was also frequently found, and was also a common limiting factor in mobilisation. In the presence of radiculopathy unresponsive to conservative management, magnetic resonance imaging was ordered with a view to possible microdiscectomy. Inflammatory joint disease, although less likely to precipitate an acute admission, was sometimes previously unrecognised and frequently undertreated. Given the need to diagnose and treat such disorders early and effectively to optimise long term outcome, ${ }^{9}$ this was of some concern.

There were some associations between rheumatic disorders and underlying medical diseases. Shoulder capsulitis tended to occur in patients with a previous cerebrovascular event, especially in association with diabetes. A single intra-articular steroid injection generally produced benefit with a transient increase in blood sugar. Gout was usually associated with diuretic treatment for heart failure, and nonsteroidal anti-inflammatory drugs were often contraindicated. Hence a trial of colchicine was frequently employed for symptomatic purposes, before the start of uricosuric therapy. The association of hypothyroidism with rheumatoid arthritis is well established and thyroid deficiency had been identified and treated in all six patients with both disorders.

Osteoporosis was especially common in the elderly, where relative immobility was a major contributing factor. However, even in patients with established fractures, prophylaxis had rarely been prescribed in spite of firm recommendations that this patient group should be treated. ${ }^{10}$

Younger patients with osteoporosis tended to have identifiable risk factors such as oral steroid exposure or chronic disease. Recent evidence for a high prevalence of osteoporosis in inflammatory bowel disease, ${ }^{11}$ asthma, ${ }^{12}$ and rheumatoid arthritis ${ }^{13}$ indicates the need for a 
high index of suspicion of osteoporosis in patients with such disorders.

The GALS technique can readily be learned in an hour and takes under two minutes to complete. It has been shown to increase confidence in locomotor examination in medical students who outperformed junior doctors not taught the technique in their examination of joints. ${ }^{14}$ It has also been shown to provide a valid and reliable measure of functional ability. ${ }^{15}$ Although the specificity of the GALS screening test falls with increasing age, its sensitivity remains high enough to justify its use, particularly in an elderly population. Abnormalities are found more frequently in the locomotor system than in any other organ complex - and many such abnormalities are amenable to therapeutic intervention. We recommend its more widespread use as a screening tool for rheumatic disease in all medical inpatients, especially where there are symptoms to suggest a positive result is likely.

1 Morbidity statistics from general practice. Third National Study Series MB5, No 1. London: HMSO, 1981-82.

2 Wright V. The epidemiology of disability. $f R$ Coll Physician Lond 1982;16:178-83.
3 Doherty M, Abawi J, Pattrick M. Audit of medical inpatient care: a cry from the joint. $\mathcal{F} R$ Coll Physicians London care: a cry from

4 Spencer MA, Dixon MS. Rheumatological features of patients admitted as emergencies to acute general medical wards. Rheumatol Rehab 1981;20:71-3.

5 Doherty M, Dacre J, Dieppe P, et al. The (GALS) locomotor screen. Ann Rheum Dis 1992;51:1165-9.

6 Jones A, Ledingham J, Regan M, et al. A proposed minimal rheumatological screening history and examination: the joint answers back. F R Coll Physicians Lond 1991;25:111-5.

7 Doherty M, Hazleman BI, Hutton CW, et al. Rheumatology Doherty $\mathrm{M}$, Hazleman $\mathrm{BI}, \mathrm{Hutton} \mathrm{CW}$, et al. Rheumatology
examination and injection techniques. Philadelphia: W B Saunders, 1992: 13-19.

8 Doherty $M$, Dawes P. Guidelines on undergraduate curriculum in the UK. Brf Rheumatol 1992;31:409-12.

9 van Jaarsveld CHM, Jacobs JWG, van der Veen MJ, et al. Aggressive treatment in early rheumatoid arthritis: a randomised controlled study. Ann Rheum Dis 2000;59:468-77.

10 Consensus Development Conference. Prophylaxis and Consensus Development Conference. Prophylaxis
treatment of osteoporosis. Am $\mathcal{F}$ Med 1991;90:107-10.

11 Scott EM, Gaywood I, Scott BB. Guidelines for osteoporosis in coeliac disease and inflammatory bowel disease. Gut 2000; suppl 1: 46.

12 Elmer K, Lapworth A, Bartholemew P, et al. Osteoporosis in asthmatics: relationship to oral steroids and male sex. Ann Rheum Dis 2000;59(suppl 1):82.

13 Haugeberg G, Uhlig T, Falch JA, et al. Bone mineral density and frequency of osteoporosis in female patients with rheumatoid arthritis: results from 394 patients in the Oslo matoid arthritis: results from 394 patients in the Oslo
County rheumatoid arthritis register. Arthritis Rheum 2000;

14 Fox RA, Dacre JE, Ingham Clark CL, et al. Impact on medical students of incorporating GALS screen teaching into medical school curriculum. Ann Rheum Dis 2000;59:668-71.

15 Plant MJ, Linton S, Dodd E, et al. The GALS locomotor screen and disability. Ann Rheum Dis 1993;52:886-90.

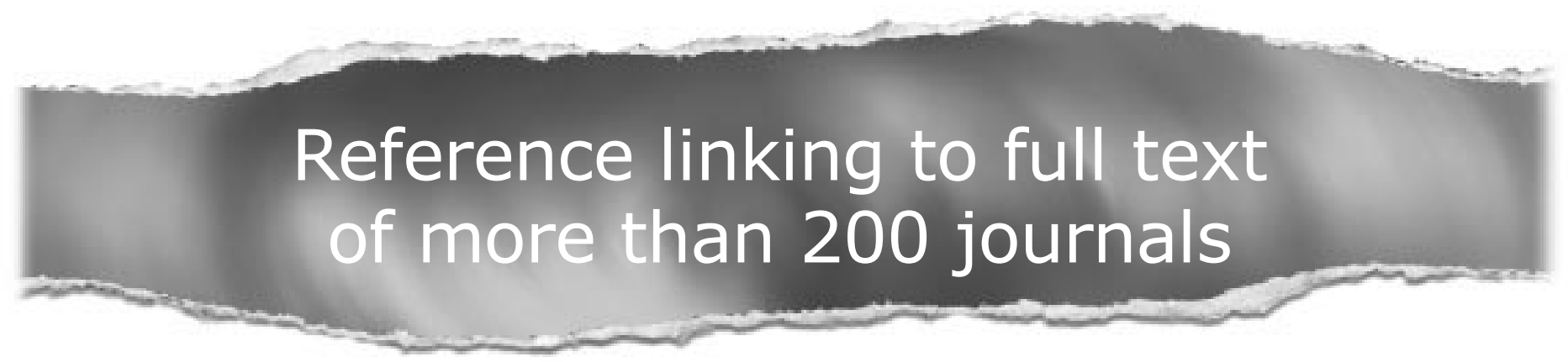

\section{Toll free links}

You can access the FULL TEXT of articles cited in Postgraduate Medical Journal online if the citation is to one of the more than 200 journals hosted by HighWire (http://highwire.stanford.edu) without a subscription to that journal. There are also direct links from references to the Medline abstract for other titles. 